\title{
Cryptogenic transient ischemic attack after nose blowing: association of huge atrial septal aneurysm with patent foramen ovale as potential cause
}

This article was published in the following Dove Press journal:

International Journal of General Medicine

I July 2013

Number of times this article has been viewed

\author{
Ulrich Lotze' \\ Uwe Kirsch' \\ Marc-Alexander Ohlow ${ }^{2}$ \\ Thorsten Scholle ${ }^{3}$ \\ Jochen Leonhardi ${ }^{3}$ \\ Bernward Lauer ${ }^{2}$ \\ Gerhard Oltmanns ${ }^{4}$ \\ Hendrik Schmidt ${ }^{5,6}$ \\ 'Department of Internal Medicine, \\ DRK Krankenhaus Sondershausen, \\ Sondershausen, Germany; \\ ${ }^{2}$ Department of Cardiology, \\ Zentralklinik Bad Berka, Bad Berka, \\ Germany; ${ }^{3}$ Institute of Diagnostic \\ and Interventional Radiology, \\ Zentralklinik Bad Berka, Germany; \\ ${ }^{4}$ Department of Internal Medicine, \\ DRK Krankenhaus Sömmerda; \\ Sömmerda, Germany; ${ }^{5}$ Department of \\ Cardiology and Diabetology, Klinikum \\ Magdeburg, Magdeburg, Germany; \\ ${ }^{6}$ Department of Internal Medicine \\ III, Martin-Luther-Univeristy Halle- \\ Wittenberg, Halle, Germany
}

\begin{abstract}
Association of atrial septal aneurysm (ASA) with patent foramen ovale (PFO) is considered an important risk factor for cardioembolism frequently forwarding paradoxical embolism in patients with cryptogenic or unexplained cerebral ischemic events. We herein describe the case of a 69 -year-old male patient reporting uncontrolled movements of the right arm due to a muscle weakness, slurred speech, and paresthesia in the oral region some seconds after he had blown his nose. These neurological symptoms had improved dramatically within a few minutes and were completely regressive at admission to our hospital about two hours later. On transesophageal echocardiography (TEE) a huge ASA associated with PFO was detected. Diagnosis of the large-sized ASA was also confirmed by cardiac magnetic resonance imaging. Due to the early complete recovery from his neurological symptoms, the patient was diagnosed with a transient ischemic attack (TIA). After nine days he was discharged in a good clinical condition under the treatment with oral anticoagulation. It is concluded that in cryptogenic or unexplained stroke or TIA TEE should always be performed to rule out ASA and PFO as potential sources for paradoxical embolism in those inconclusive clinical situations.
\end{abstract}

Keywords: congenital cardiac abnormality, atrial septal aneurysm, nose blowing, paradoxical embolism, patent foramen ovale, transient ischemic attack

\section{Introduction}

Association of atrial septal aneurysm (ASA) with patent foramen ovale (PFO) is considered an important risk factor for cardioembolism frequently forwarding paradoxical embolism in patients with cryptogenic or unexplained cerebral ischemic events. $^{1-4}$

\section{Case presentation Symptoms and history}

In February 2012, a 69-year-old man was referred to our emergency department. He had uncontrolled movements of the right arm not corresponding to fasciculations, but primarily due to muscle weakness, slurred speech, and paresthesia in the oral region some seconds after he had blown his nose. All neurological symptoms dramatically improved again after a few minutes and were not detectable any more at admission to our hospital about two hours later. Previous diagnoses were arterial hypertension, non insulin-dependent diabetes mellitus, paroxysmal atrial fibrillation, stenting of the left internal carotid artery, inferior wall myocardial infarction, and chronic obstructive lung disease.
Correspondence: Ulrich Lotze Department of Internal Medicine, DRK Krankenhaus Sondershausen, Hospitalstr 2,

D-99706 Sondershausen, Germany

Tel +49363267 II07

Fax +493632 67 I019

Email lotze@sdh.drk-tb.de 


\section{Basic cardiologic findings}

Further cardiologic examination showed no significant findings at heart auscultation. The 12 lead electrocardiogram (Bioset 8000, Hörmann Medizintechnik Zwönitz GmbH, Zwönitz, Germany) revealed a sinus rhythm with a normal electrical axis of the heart of $30^{\circ}-60^{\circ}$ on the frontal plane and neither repolarization disorders nor bundle branch block.

\section{Imaging techniques}

A cranial computed tomography (CT; SOMATOM Emotion 6, Siemens AG, Healthcare Sector, Erlangen, Germany) revealed no acute ischemia or hemorrhage, but an old small lacunary lesion on the left side of the cerebellum.

Transthoracic echocardiography (TTE; Vivid 7 Dimension, GE Wingmed Ultrasound A/S, Horten, Norway), showed regular size of all four chambers, normal left ventricular ejection fraction of $60 \%$ with hypokinesia inferior and posterior due to an old myocardial infarction, and diastolic dysfunction at grade I. Minimal mitral and tricuspid regurgitation associated with a maximum transtricuspid pressure gradient of $30 \mathrm{mmHg}$ were found. From the apical four-chamber view a huge atrial septal aneurysm (ASA) with a bulging into the right atrium (RA) of $18 \mathrm{~mm}$ and a base width of $26 \mathrm{~mm}$, associated with a small left-to-right shunt as assessed by color and pulsed-wave (PW) Doppler examination (Vivid 7 Dimension, GE Wingmed Ultrasound A/S, Horten, Norway), was seen (Figure 1A and B). Subsequently, a transesophageal echocardiography (TEE) was performed to confirm the association of the two anatomical abnormalities involving the interatrial septum (IAS).

On TEE, an ASA with a prominent bulging into the RA of a size similar to that detected by TTE could be confirmed (Figure 2A). Moreover, a small PFO with approximately $1.5 \mathrm{~mm}$ of separation between septum primum and secundum characterized by spontaneous left-to-right shunt (Figure 2B) and right-to-left shunt during Valsalva maneuver (Figure 2C) was demonstrated. No other cardiac anatomical abnormality
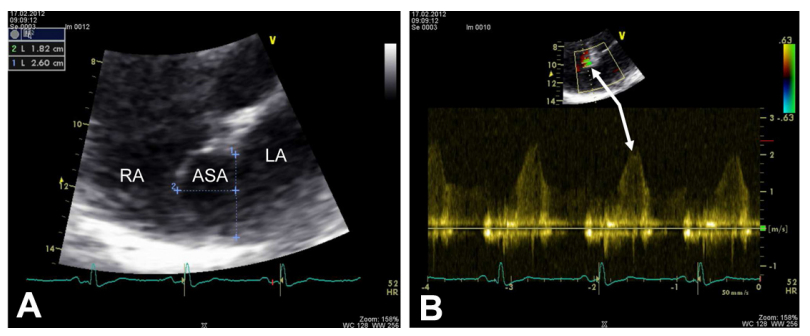

Figure I Transthoracic echocardiogram. (A) Four-chamber view with the use of zoom function demonstrates an ASA with a pronounced protrusion into the RA of $18 \mathrm{~mm}$ and a base width of $26 \mathrm{~mm}$. (B) Color and PW Doppler echocardiographic examination reveals a small left-to-right shunt through a small PFO (white arrows). Abbreviations: ASA, atrial septal aneurysm; LA, left atrium; PFO, patent foramen ovale; RA, right atrium.

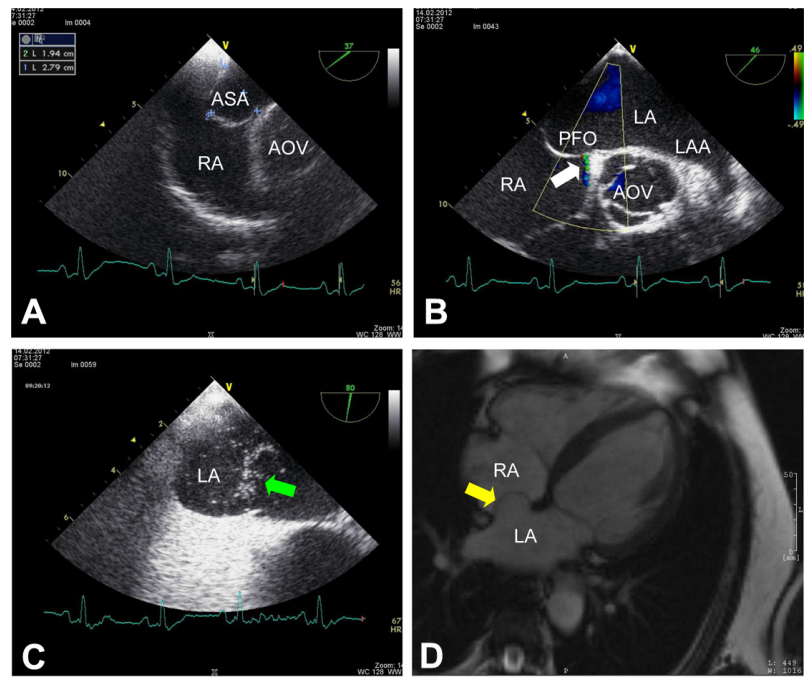

Figure 2 Transesophageal echocardiogram and cardiac magnetic resonance imaging (MRI). (A)Transesophageal short-axis view at $37^{\circ}$ confirms the giant ASA with an excursion into the RA of $19 \mathrm{~mm}$ and a base diameter of $28 \mathrm{~mm}$. (B) Transesophageal short-axis view $\left(46^{\circ}\right)$ shows a left-to-right shunt through a small PFO (white arrow) as detected by color Doppler. (C) Using agitated gelafundin (4\%) intravenously some microbubbles (green arrow) can be demonstrated in LA during Valsalva maneuver indicating a right-to-left shunt through the small PFO in case of elevated intrathoracic pressure (transesophageal short-axis view at $80^{\circ}$ ). (D) Cardiac MRI (transverse plane) also reveals a huge ASA with protrusion into the RA (yellow arrow).

Abbreviations: AOV, aortic valve; ASA, atrial septal aneurysm; LA, left atrium; LAA, left atrial appendage; PFO, patent foramen ovale; RA, right atrium.

was found. Cardiac magnetic resonance imaging (MRI; MAGNETOM Avanto 1.5T, Siemens AG, Healthcare Sector, Erlangen, Germany) also revealed a huge ASA (Figure 2D).

On color Doppler and duplex ultrasonography, a general atherosclerosis and plaques were found in the brain-supplying arteries, and a mild-to-moderate stenosis of the external carotid artery on the left side, but no in-stent restenosis of the left internal carotid artery could be detected. Furthermore, Doppler ultrasound examination of the lower extremities demonstrated no deep vein thrombosis.

\section{Diagnosis and clinical course}

Since the neurological symptoms improved dramatically within a few minutes and had completely regressed after two hours, the patient was diagnosed with a transient ischemic attack (TIA). Therefore, an intracranial MRI including an angiographic study was not performed in addition to the CT scan of the brain. The possibility of another paroxysmal neurological disease such as epilepsy was discussed and excluded by a neurological consultation. Taking into account the association of the combined occurrence of ASA and PFO with a TIA, oral anticoagulation $(\mathrm{OAC})$ with phenprocoumon was initiated in our patient. The small PFO was not considered absolutely liable for interventional treatment with a septal occluder device and the patient was discharged after nine days in a good clinical 
condition. At a follow-up visit after eight months the patient had not experienced any further neurological symptoms during common daily activities under ongoing OAC.

\section{Discussion}

Given the patient's neurological symptoms after blowing his nose and the association of ASA with PFO it can be assumed that the otherwise unexplained TIA primarily was caused by a paradoxical embolism due to right-to-left shunting in our patient. According to the neurological consultation epilepsy initially considered as differential diagnosis was not confirmed. Moreover, the cranial CT findings including missing acute ischemia or hemorrhage, respectively, and the small old intracerebellar lacunary lesion hardly caused by an embolic event, but primarily by local microangiopathic disorders ${ }^{5,6}$ are well compatible with the clinical diagnosis of an acute TIA.

ASA and PFO are rare, but well known cardiac abnormalities both previously described in autopsy studies, ${ }^{7,8}$ and nowadays more and more are found by TTE and TEE, especially in patients with preceding ischemic cerebral events. ${ }^{1-4}$

The prevalence of ASA in the general population varies between $1 \%$ as verified in a large autopsy series ${ }^{7}$ and $2.2 \%$ as detected by TEE, with a base width of at least $15 \mathrm{~mm}$ and an excursion of at least $15 \mathrm{~mm}$ into the right or left atrium. ${ }^{3}$

In patients with stroke or TIA, however, ASA has been reported in $7.9 \%-46 \%$ depending on the cut-off of the excursion of $\geq 10$ or $\geq 15 \mathrm{~mm}$, respectively, into either the right or left atrium and on whether all cerebral ischemic events or only cryptogenic stroke or TIA, due to possible cardiogenic embolism were included in the evaluation. ${ }^{1-3}$

As far as the pathophysiological mechanism of this 'saccular' malformation is concerned, a particular congenital morphology of the fossa ovalis region or interatrial pressure differences may be predisposing factors for the development of an ASA. ${ }^{7,8}$ Furthermore, a disorder of the cardiac connective tissue may also play a significant role in the pathogenesis of this deformity of the IAS. ${ }^{9}$

The additionally detected PFO with spontaneous left-toright shunt and a right-to-left shunt under Valsalva maneuver is an embryonic remnant and found in approximately one quarter of all adults ${ }^{10}$ and in $15 \%-56.3 \%$ of patients with stroke or TIA, respectively, or suspected cardioembolic cerebral event. ${ }^{1,2,4}$ Furthermore it is interesting to mention that a PFO can be found in up to $40 \%$ of younger patients with otherwise cryptogenic stroke. ${ }^{11}$ Association of ASA with PFO was described in $50 \%-72 \%$ of the cases in pathological studies ${ }^{7,8}$ and in 34\%-56\%, as detected by TEE, of patients with possible cardiogenic cerebral embolism..$^{2-4}$
However, in another large clinical series ASA and PFO as detected by TTE were the source of embolism in only two of 402 consecutive patients with ischemic cardioembolic stroke $^{12}$ highlighting the relevance of the present case and the importance to carefully look for ASA and PFO in patients with cryptogenic stroke or TIA.

The detection of these two cardiac abnormalities is frequently associated with a preceding cerebral ischemic event ${ }^{1-4}$ and therefore considered a strong predictor of stroke. ${ }^{1}$

In stroke patients with ASA and PFO, however, several mechanisms leading to the cerebral ischemic event can be discussed. First of all, paradoxical embolism through the PFO after Valsalva maneuver-like procedures such as blowing the nose, as in our case, has been commonly thought to be responsible for the cerebral ischemic event. ${ }^{1}$ But according to pathological findings by Silver and Dorsey ${ }^{7}$ and Topaz et $\mathrm{l}^{8}$ direct embolization from thrombi developed within the ASA or embolization through multiple perforations of a so called cribriform $\mathrm{ASA}^{13}$ can also be taken into consideration as further sources of stroke. ASA may also contribute to paradoxical embolism by channeling the blood flow from the inferior vena cava towards a PFO, and thus forwarding a right-to-left shunt. ${ }^{14}$ Furthermore, mitral valve prolapse ${ }^{9}$ and atrial arrhythmias ${ }^{1}$ are additional potential causes for cardioembolism in stroke patients with ASA.

Although the patient has several risk factors such as hypertension, diabetes mellitus, paroxysmal atrial fibrillation, carotid stenting on the left side and an old inferior wall myocardial infarction for having a stroke or a TIA and nose blowing is a common procedure during daily life even in patients with ASA and PFO, the very close temporal relation between nose blowing, a Valsalva-like maneuver, and the neurological symptoms corresponding to a TIA primarily indicate that in this clinical situation described herein our patient had suffered a paradoxical embolic cerebral event.

Finally, it has to be conceded that the source of the suggested paradoxical embolism leading to a TIA directly after nose blowing could not be clearly discovered in our case, but at least a slight deep vein thrombosis of the lower extremities that could not be detected later any more may be primarily thought to be a common and the most probable cause for the preceding ischemic cerebral event.

Since the TIA in our patient was probably his first typical cardioembolic ischemic cerebral event, interventional correction of the small PFO with a diameter of $1.5 \mathrm{~mm}$ was not considered absolutely necessary, but in case of a recurrent stroke or TIA under OAC, treatment with a septal occluder 
device will be indicated ${ }^{15}$ keeping in mind that patients with ASA and interatrial shunt have an increased risk of recurrent stroke or TIA. ${ }^{16}$

In conclusion, association of ASA with PFO should always be taken into consideration as a potential cause for paradoxical embolism in cryptogenic stroke or TIA, especially after a Valsalva-like maneuver such as nose blowing. Therefore, TEE should be performed in all clinical inconclusive situations with suspected cardioembolism to safely recognize each of those two structural abnormalities of the IAS and to initiate the optimal therapeutic regimen for secondary prevention.

\section{Consent}

Written informed consent was obtained from the patient in order to be allowed to publish this case report.

\section{Disclosure}

The authors report no conflicts of interest in this work.

\section{References}

1. Mas JL. Patent foramen ovale, atrial septal aneurysm and ischaemic stroke in young adults. Eur Heart J. 1994;15(4):446-449.

2. Mügge A, Daniel WG, Angermann C, et al. Atrial septal aneurysm in adult patients. A multicenter study using transthoracic and transesophageal echocardiography. Circulation. 1995;91(11):2785-2792.

3. Agmon Y, Khandheria BK, Meissner I, et al. Frequency of atrial septal aneurysms in patients with cerebral ischemic events. Circulation. 1999;99(15):1942-1944.

4. Serafini O, Misuraca G, Greco F, Bisignani G, Manes MT, Venneri N. Prevalence of structural abnormalities of the atrial septum and their association with recent ischemic stroke or transient ischemic attack: echocardiographic evaluation in 18631 patients. Ital Heart J Suppl. 2003;4(1):39-45.
5. Ringelstein EB, Koschorke S, Holling A, Thron A, Lambertz H, Minale C. Computed tomographic patterns of proven embolic brain infarctions. Ann Neurol. 1989;26(6):759-765.

6. Leistner S, Huebner N, Faulstich A, et al. +Increased prevalence of microangiopathic brain lesions among siblings of patients with lacunar stroke. A prospective multicenter study. Eur Neurol. 2008;59(3-4): 143-147.

7. Silver MD, Dorsey JS. Aneurysms of the septum primum in adults. Arch Pathol Lab Med. 1978;102(2):62-65.

8. Topaz O, Edwards JE, Bojack-Mackey S, Titus JL. Aneurysm of fossa ovalis in adults: a pathologic study. Cardiovasc Pathol. 2003;12(4): 219-225.

9. Rahko PS, Xu QB. Increased prevalence of atrial septal aneurysm in mitral valve prolapse. Am J Cardiol. 1990;66(2):235-237.

10. Movsowitz C, Podolsky LA, Meyerowitz CB, Jacobs LE, Kotler MN. Patent foramen ovale: a nonfunctional embryological remnant or a potential cause of significant pathology? J Am Soc Echocardiogr. 1992;5(3):259-270.

11. Arboix A, Alió J. Acute cardioembolic stroke: an update. Expert Rev Cardiovasc Ther. 2011;9(3):367-379.

12. Pujadas Capmany R, Arboix A, Casañas-Muñoz R, Anguera-Ferrando N. Specific cardiac disoders in 402 consecutive patients with ischaemic cardioembolic stroke. Int J Cardiol. 2004;95(2-3):129-134.

13. Vistarini N, Aiello M, Viganò M. Cribriform atrial septal aneurysm. J Am Coll Cardiol. 2009;53(25):2404.

14. De Castro S, Cartoni D, Fiorelli M, et al. Morphological and functional characteristics of patent foramen ovale and their embolic implications. Stroke. 2000;31(10):2407-2413.

15. Meier B, Frank B, Wahl A, Diener HC. Secondary stroke prevention: patent foramen ovale, aortic plaque, and carotid stenosis. Eur Heart J. 2012;33(6):705-713, 713a, 713b.

16. Comess KA, DeRook FA, Beach KW, Lytle NJ, Golby AJ, Albers GW. Transesophageal echocardiography and carotid ultrasound in patients with cerebral ischemia: prevalence of findings and recurrent stroke risk. J Am Coll Cardiol. 1994;23(7):1598-1603.
International Journal of General Medicine

\section{Publish your work in this journal}

The International Journal of General Medicine is an international, peer-reviewed open-access journal that focuses on general and internal medicine, pathogenesis, epidemiology, diagnosis, monitoring and treatment protocols. The journal is characterized by the rapid reporting of reviews, original research and clinical studies across all disease areas.

\section{Dovepress}

A key focus is the elucidation of disease processes and management protocols resulting in improved outcomes for the patient.The manuscript management system is completely online and includes a very quick and fair peer-review system. Visit http://www.dovepress.com/ testimonials.php to read real quotes from published authors. 